\title{
Numerical models development for unidirectional air flow diffusers with lobed and circular orifices
}

\author{
Laurentiu Tacutu ${ }^{1,2}$, Ilinca Nastase $^{1,2}$, Florin Bode ${ }^{1,2,3}$, Cristiana Croitoru ${ }^{1,2}$, Catalin Lungu ${ }^{2}$ \\ ${ }^{1}$ CAMBI Research Center, Technical University of Civil Engineering Bucharest, 021414 Bucharest, Romania \\ ${ }^{2}$ Technical University of Civil Engineering Bucharest, Faculty of Building Services, 021414 Bucharest, Romania \\ ${ }^{3}$ Technical University of Cluj-Napoca, Department of Mechanical Engineering, 400020 Cluj-Napoca, Romania
}

\begin{abstract}
In order to achieve more realistic boundary conditions on the inlet of a ventilation system it is necessary to study the influences of the air diffuser orifices geometry on the airflow distribution in the enclosure. Integrating these orifices directly in a real scale air diffuser for a numerical study will result in a huge computational grid which will translate in huge computational resources and a much larger calculation time. The solution, in this case, was the numerical simulation of the airflow through small parts of the studied air diffuser. Later, the numerical results will be implemented as boundary conditions in the unidirectional diffuser of a numerical simulation that represents a real scale operating room (OR). In the current study two diffusers with different orifices were studied, one having circular (,O”) and the other one lobbed (,+") orifices. The initial numerical model had 25 orifices on the diffuser, but because of the very large numerical grid resulted for the initial meshes ( $>35$ million tetrahedral cells), a solution with only 4 orifices was chosen for this study. A mesh independency study was made for these two types of air diffusers. The numerical studies were made using RANS method, with SST k- $\omega$ turbulence model in steady state conditions. The numerical results obtained with the first step models showed very good agreement with the PIV stereoscopic experimental measurements.
\end{abstract}

\section{Introduction}

When performing airflow analyses on a ventilation system in an enclosure it is necessary to study which are the implications of the geometry of the orifices of the diffuser have on the airflow. This approach can give a deeper understanding in the studies carried out in the field of air distribution and the predicted results can be closer to the real in-situ developing flows. There are studies which state that the orifices of a diffuser can be used as a passive control method of the air jet type flows to improve the mixing of the injected air with its ambient air [1-5]. The mixing process is closely correlated to the turbulence transition [6], while the geometry and the initial flow perturbation significantly affect its generation and transition. For residential purposes, they can have direct implications regarding the thermal comfort of the occupants in the room, the dispersion of pollutants or the energy consumptions of the HVAC unit. The main disadvantage in studying the implementation of small geometries like orifices in the air diffusers is that they lead to a huge number of elements which would require high computing resources and a large amount of time to solve the calculations. The number of the mesh elements will increase in the case of a numerical study in which several diffusers or diffusers with large surfaces are studied. This paper presents a study for two air diffusers with two different orifice geometries, a lobed (,,") and a circular one („O), by means of numerical and experimental measurements. The geometries were studied in previous research but at a smaller sizes [3, 7-10]. The aim of the study was to analyse the fluid jets from these two types of orifices and how they interact with each other at different distances from the diffuser. The results from the numerical case, after validating them against experimental measurements, were used as a boundary conditions in the larger numerical case that represents an operating room (OR) with a unidirectional air flow (UAF) plenum, also called a laminar air flow (LAF) plenum. Implementing them in this larger numerical case will allow to study the air distribution in the room and to make comparison between the two types of orifices. This study addresses the idea of a passive control $[11,12]$ for a better distribution of the air in the room, achieving also a better thermal stratification, having the potential to improve the energy consumption.

\section{Methods}

A mesh independency study was made for the numerical approach, while experimental measurements with PIV Stereoscopic were carried for validating the numerical study. Initially, a $100 \times 100 \mathrm{~mm}$ grid was meant to be used, resulting a total of 25 orifices, but at pour mesh quality the number of elements easily exceeded 35 million tetrahedral elements. Given this fact, the estimation was that for a high-quality numerical grid, the computational

\footnotetext{
* Corresponding author: ilinca.nastase $@$ gmail.com
} 
resources we have access would have been overcome. In order to reduce the number of elements we reduced the number of studied orifices to 4 . In this way we can still analyse the interaction between the air jets through the orifices, and we can have a number of elements at a highquality mesh that can be numerical simulated in a decent amount of time. The entire real geometry of the studied model consists in an aluminium plate of $1.5 \mathrm{~mm}$ thickness, which represents the diffuser, with 4 orifices, and has a total domain of $240 \mathrm{~mm}$ in length, with 20 equivalent diameters $(\mathrm{De})$ of the orifice after the diffuser $(\approx 150 \mathrm{~mm})$. A total of 4 different meshes were analysed for both orifices. For the circular orifices case (,O”) the meshes were between $4.6 \div 20.8$ million, while for the lobed orifices case $(,+$,$) the meshes were between 8.6 \div 35.5$ million tetrahedral elements. The study was conducted in Ansys 18.2, using Space Claim for modelling and Fluent for the numerical simulation, while the post-processing was made in Tecplot 360 . The geometry was made in Solidworks and imported in Ansys Workbench. Boundary conditions were imposed, coupled pressure-based solver was used and hybrid initialization, SST k- $\omega$ turbulence model was used, and the fluid was water. The experiments were made in water, thus the initial numerical cases used also water in order to validate them against experimental data. A simplified hypothesis was disabling gravity because its influence is very small, infinitesimal, since the impulse forces are much higher than the gravity force (Archimedes forces) in this case. In Figure 1 we can see a sketch with the numerical study that represents an OR and the boundary conditions, from the orifices, that will be implemented. The geometry of the OR will have two surgeons and a patient, a general ventilation system, the unidirectional air flow diffuser, and a local ventilation system. The exhaust will be made by corner outlets, two on each corner.

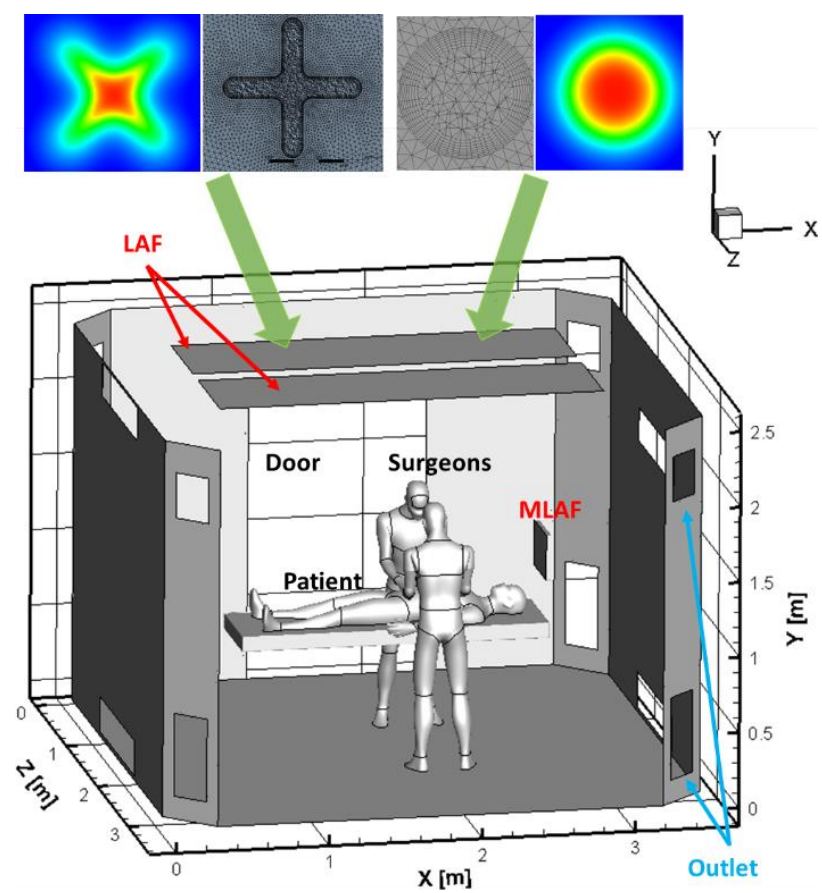

Fig. 1. Operating room geometry and the boundary conditions with orifices (lobed and circular)

\section{Numerical simulations}

First type of geometry represents a baseline that is used nowadays in air terminals devices, which has a circular shape (,O”). The second geometry, named lobed (,,+"), is used more in a specific field, aeronautics and combustion [13-15]. Both shapes with their dimensions can be seen in the Figure 2.
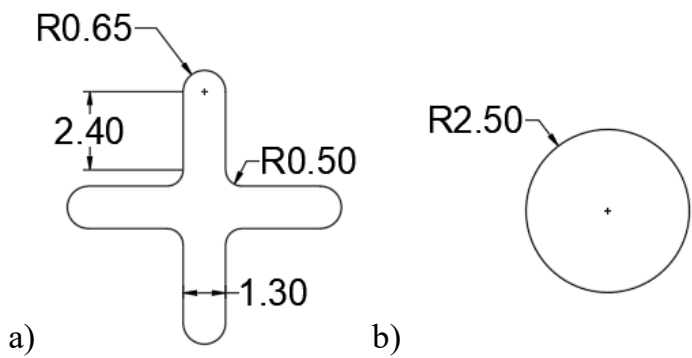

b)

Fig. 2. The orifices with technical details of the geometries: a) lobed or ,+”; b) circular or „O”

The 3D geometries were made in SolidWorks and imported to DesignModeler from Ansys Workbench. Ansys Workbench 18.2 was used for creating the numerical model. The initial numerical case was created according with the aluminium plates (diffuser) that were made for the PIV measurements (Figure 3). Because of the high number of elements resulted when creating the mesh for the diffuser with lobed orifices $(\geq 35$ million tetrahedral elements), we choose a 4 orifices diffuser for using in the numerical simulated case (Figure 4).

a)
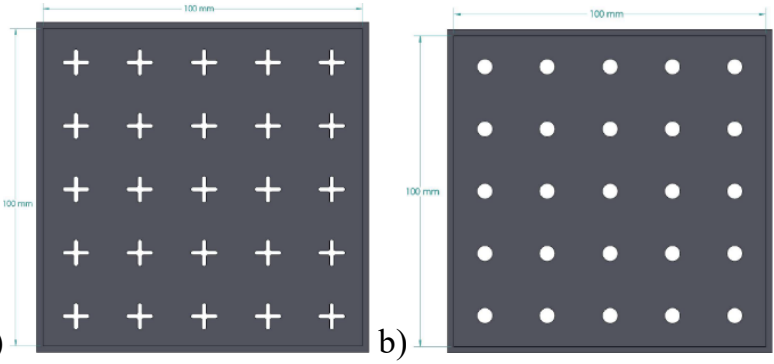

Fig. 3. Diffusers with 25 orifices of $100 \times 100 \mathrm{~mm}$ : a) lobed or „,"; b) circular or „O”

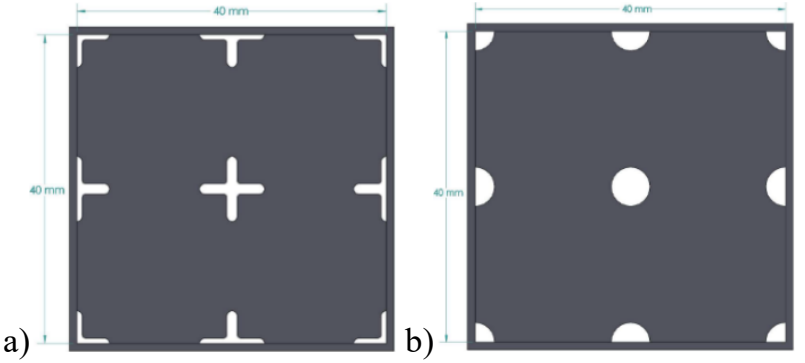

Fig. 4. Diffusers with 4 orifices of $40 \times 40 \mathrm{~mm}$ : a) lobed or ,+”; b) circular or „O”

The domain of the numerical case (Figure 5) it's in the form of a cuboid that has a length of $240 \mathrm{~mm}$, with $40 \times 40 \mathrm{~mm}$ height and width, with 20De after the diffuser $(\approx 150 \mathrm{~mm})$ in order to study the fluid flow of the jets and their interactions. 

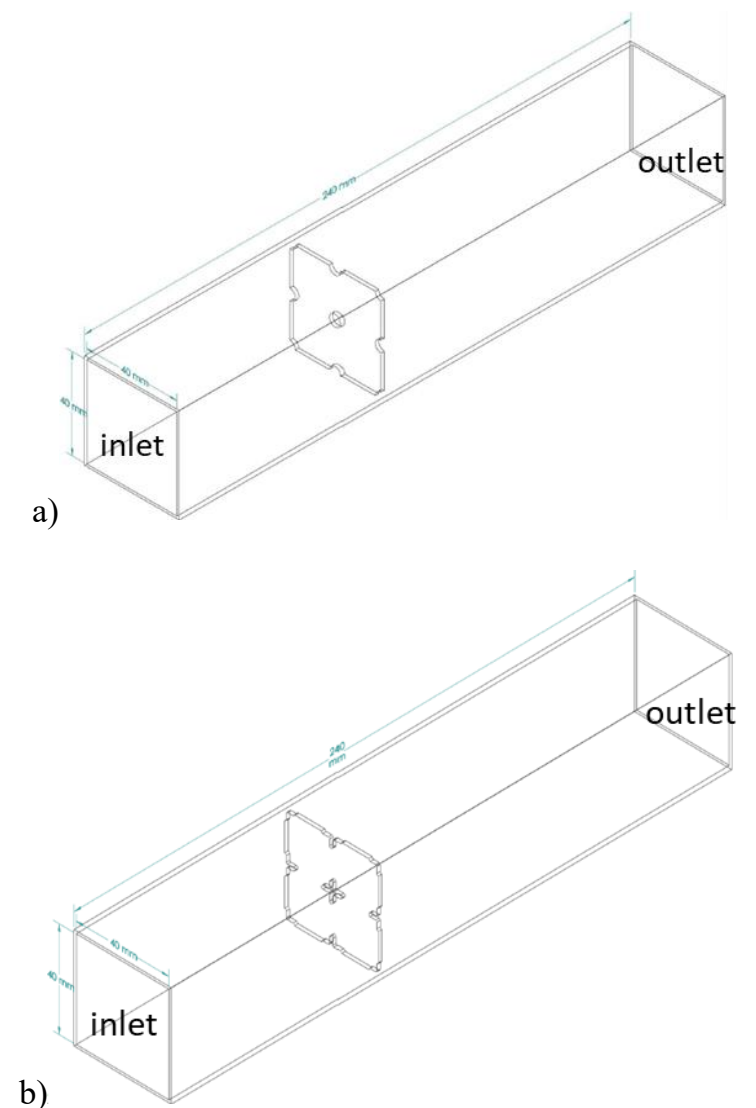

Fig. 5. The domain of the numerical cases with diffusers of 4 orifices: a) circular; b) lobed

For a better control of the cell size in the interested areas, before and after the orifices, ,body of influence” option was used in those regions. In this way we added more elements in zones where we encountered large gradients of velocity. The studied domain with this option can be seen in Figure 6.

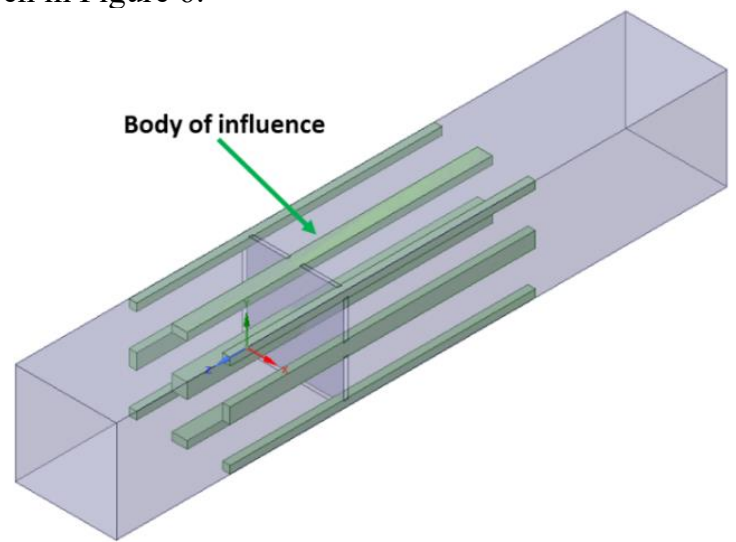

Fig. 6. The domain of the numerical cases with diffusers and the „body of influence” option used

The meshes generated for this two studied case have the following sizes:

- Small diffuser with lobed orifices $(,,+"): 8.6,13.5$, 18.8 and 35 million tetrahedral elements, respectively 1.8, 2.6, 3.6 and 6.4 million polyhedral elements;

- Small diffuser with circular orifices („O”): 4.6, 7.4, 10.8 and 20.8 million tetrahedral elements, respectively $870 \mathrm{k}, 1.3,1.6$ and 3.7 million polyhedral elements.
Front sections from the mesh of a lobed diffuser centered on the interested domain is presented in the Figure 7.

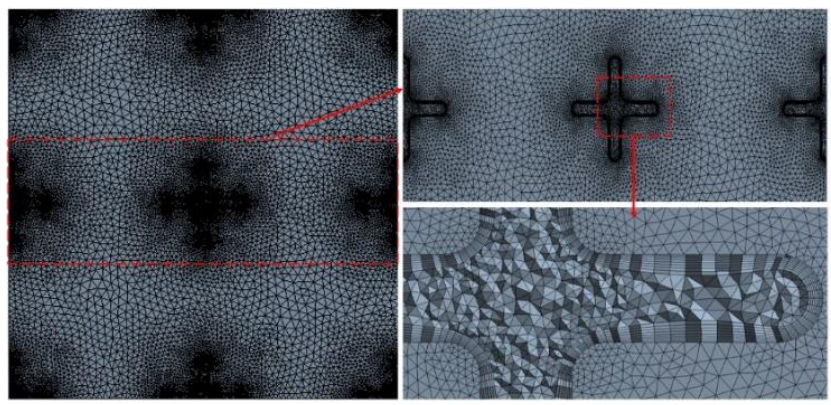

Fig. 7. Front section of the mesh from the lobed diffuser in the interest domain

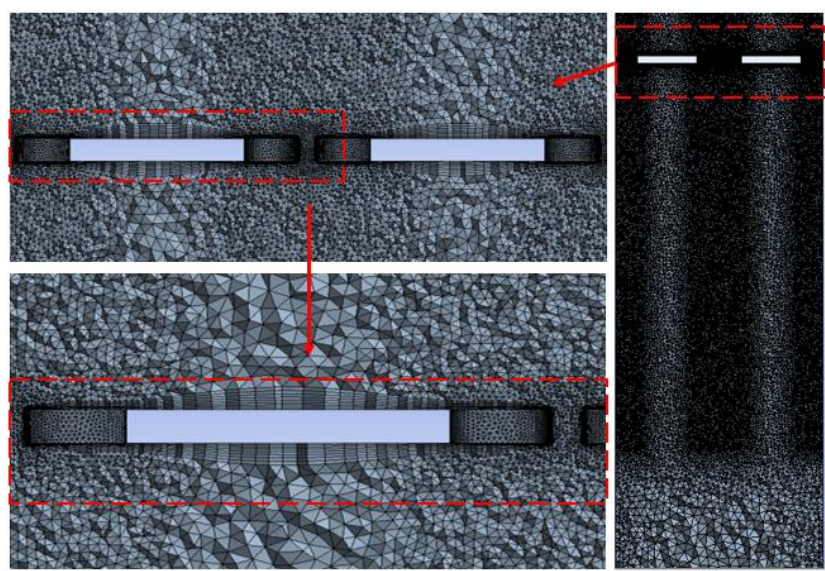

Fig. 8. Cross section of the mesh from the lobed diffuser in the interest domain

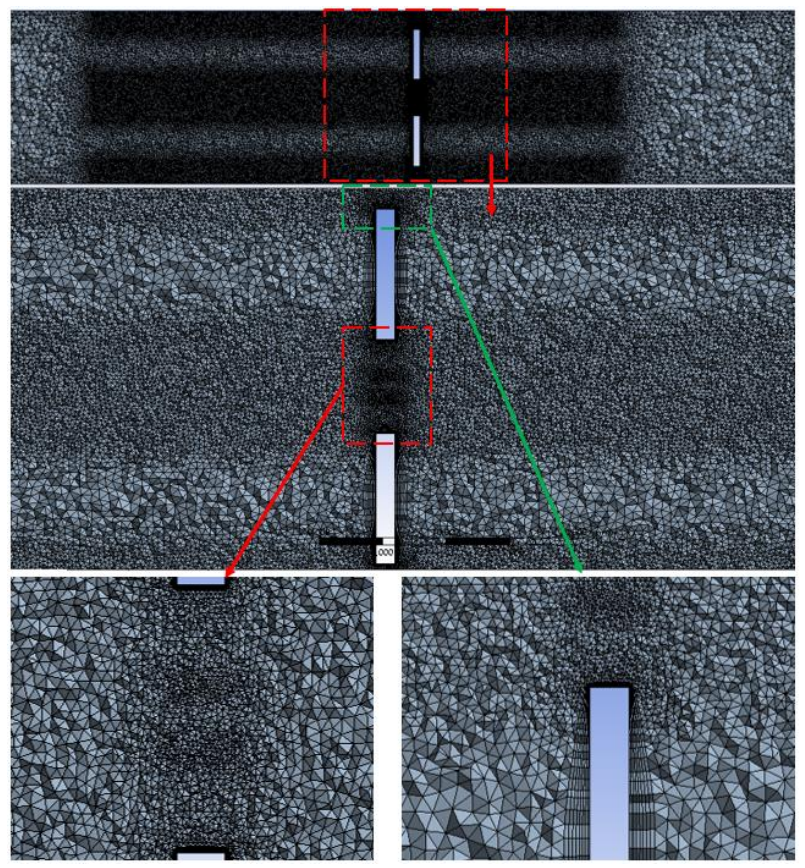

Fig. 9. Sagittal section of the mesh from the lobed diffuser with the interested domain

Cross and sagittal sections of a lobed diffuser can be seen in Figure 9 and 10. In these pictures it can be seen the differences in cell size using the option used, „body of influence", the way of generating the boundary layer and 
the number of boundary layers. A maximum number of 8 boundary layers was generated. The growth rate was set at 1.1, while the layer height was generated in accordance with the cell size in the respective region.

Front sections from all 4 meshes of the lobed diffuser, centered on the interested domain, can be seen in Figure 10. This figure shows the differences in cell dimensions of a lobed orifice between the numerical cases studied that had this configuration.
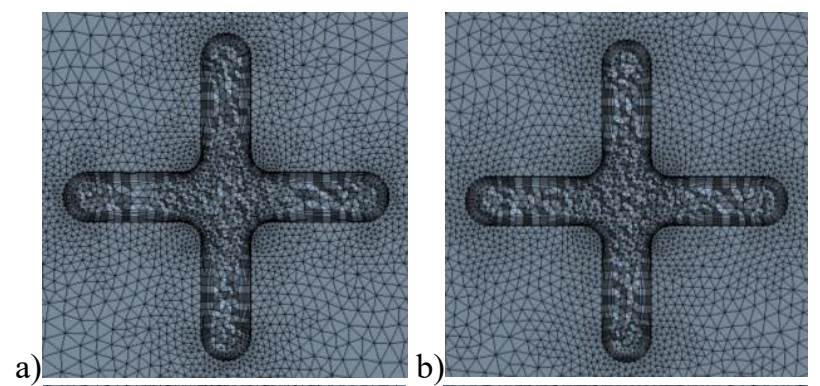

a)
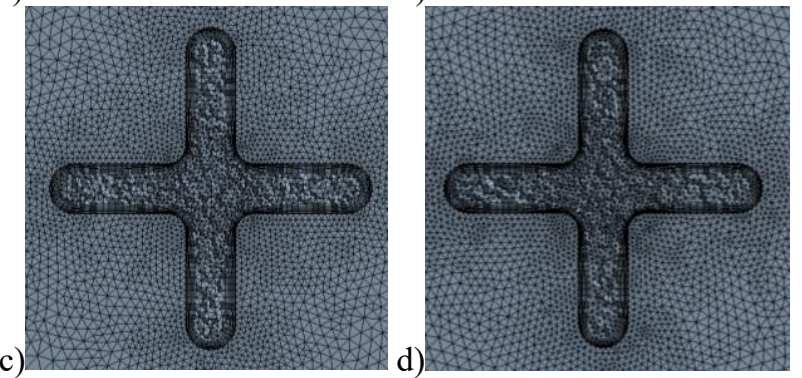

Fig. 10. Front sections of the mesh from the lobed diffuser for the cases: a) 8.6 ; b) 13.5 ; c) 18.8 ; d) 35 [million tetrahedral elements]

The meshes for the diffuser with circular orifices didn't present the same complexity as for the lobbed diffuser, resulting also in a much lower number of cells. A front section of the whole diffuser (plate) with circular orifices is presented in the Figure 11.

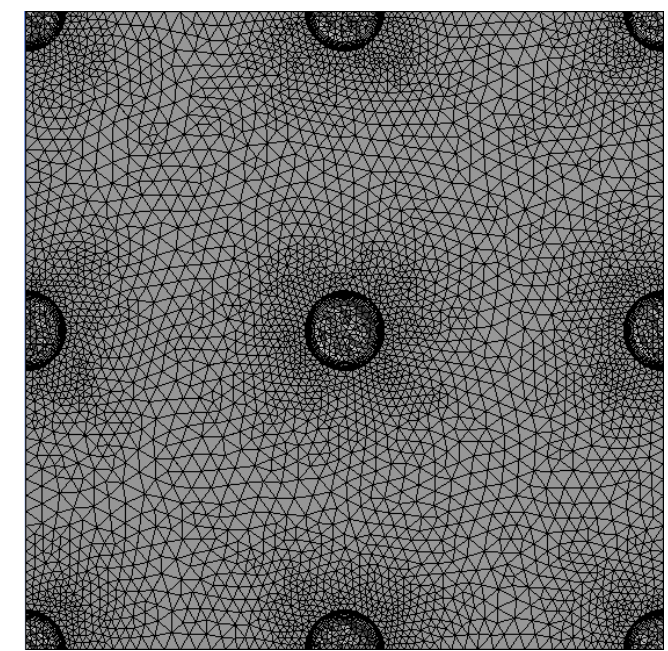

Fig. 11. Front section of the mesh from the circular diffuser with the interested domain

The calculations were made using RANS equations, in steady-state conditions. The turbulence model used was SST k-omega, with Pressure-Velocity Coupling type Coupled and Least Squares Cell Based, Second Order or
Second Order Upwind. The initialization was set to hybrid. Water was used as a fluid, without the gravity force. The convergence set for all residuals was $10^{\wedge}(-6)$. All the calculations were made in isothermal conditions $\left(20^{\circ} \mathrm{C}\right.$ as boundary conditions on the walls and for the fluid). The inlet for the fluid was set as the entire surface of the back wall while the outlet was set as the entire surface of the front wall (Figure 5).

\section{Experimental studies}

The experimental studies were carried out in isothermal conditions, $\approx 20^{\circ} \mathrm{C}$, in an enclosure filled with water (Figure12) were water jets were formed by circulating the water with a pump.
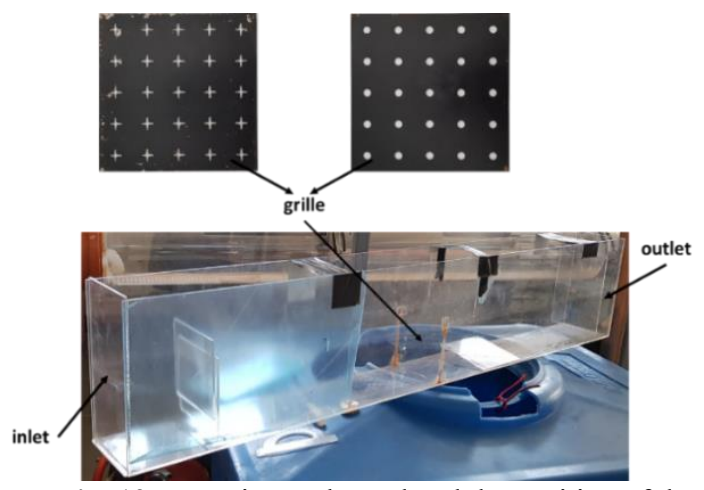

Fig. 12. Experimental stand and the position of the diffusers

The water was seeded with small fluorescent particles of $\mathrm{D} 50=20 \mu \mathrm{m}$. The diffusers (plates) were painted in black in order to avoid reflections from them when measured with the PIV system. Because the measurements were made in Stereoscopic PIV, it was able to capture the three components of the velocity filed ( $\mathrm{U}, \mathrm{V}, \mathrm{W}$ or $\mathrm{Vx}, \mathrm{Vy}, \mathrm{Vz}$ ). The time interval between two laser beams was set at 250 $\mu$ s and a number of 500 frames were captured during each season of measurements. Cross correlation analyses of the velocity vectors were made with the software of the PIV system. The PIV measurement were made in three vertical planes which are shown in Figure 13. The data was after post-process with Tecplot by Kriging interpolation, resulting a 3D volume (Figure 20) from the three planes of measurements (Figure 13).

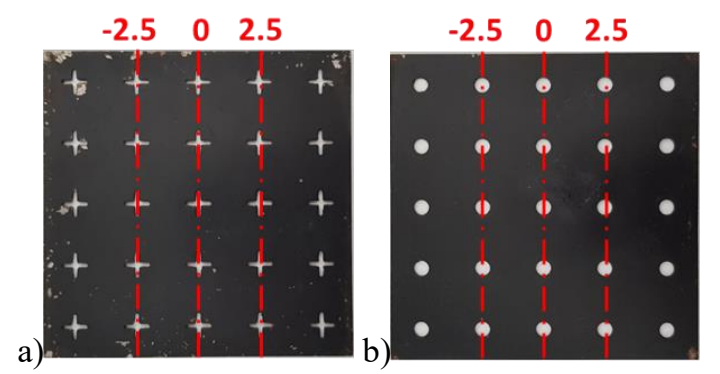

Fig. 13. The planes of the PIV measurements: a) lobbed diffuser; b) circular diffuser

\section{Results}

A comparison of velocity profiles from the center of the orifices were made for all the 4 cases of each orifice for analyzing the mesh influence (Figure 14). 


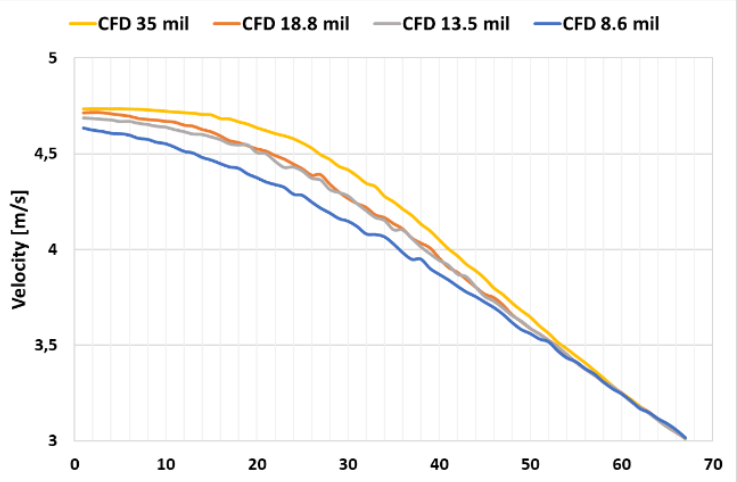

a)

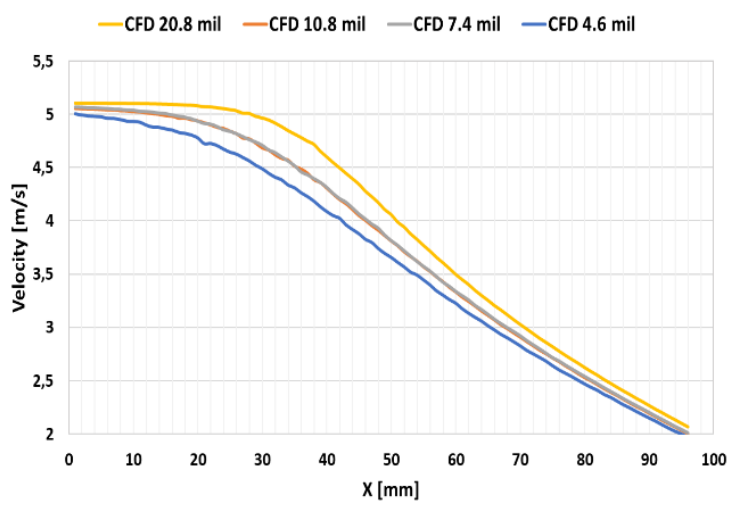

b)

Fig. 14. Velocity fields comparison for the numerical cases: a) lobbed orfice; b) circular orfice

For the lobbed diffuser resulted that the numerical cases with 18.8 and 13.5 million cells give the same profiles of velocity while 35 and 8.6 million cells give maximum and minimum profiles. The same remark is observed for the circular diffusers. Comparations of velocity profiles were made at different distances corresponding to equivalent diameters. The distance considered are represented in Figure 15. For one equivalent diameter (1De) the distance is $7,5 \mathrm{~mm}$ and this value multiplies in accordance with the respective distance.

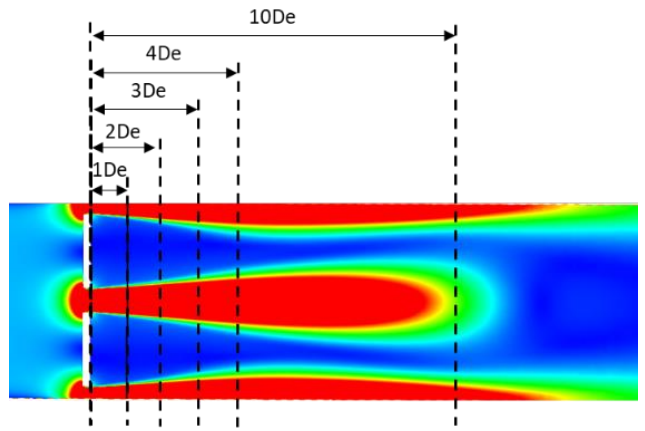

Fig. 15. Representing distance at different equivalent diameters $(\mathrm{De})$

Velocity profiles are presented in Figure 16 and 17 at different distances corresponding to equivalent diameters.

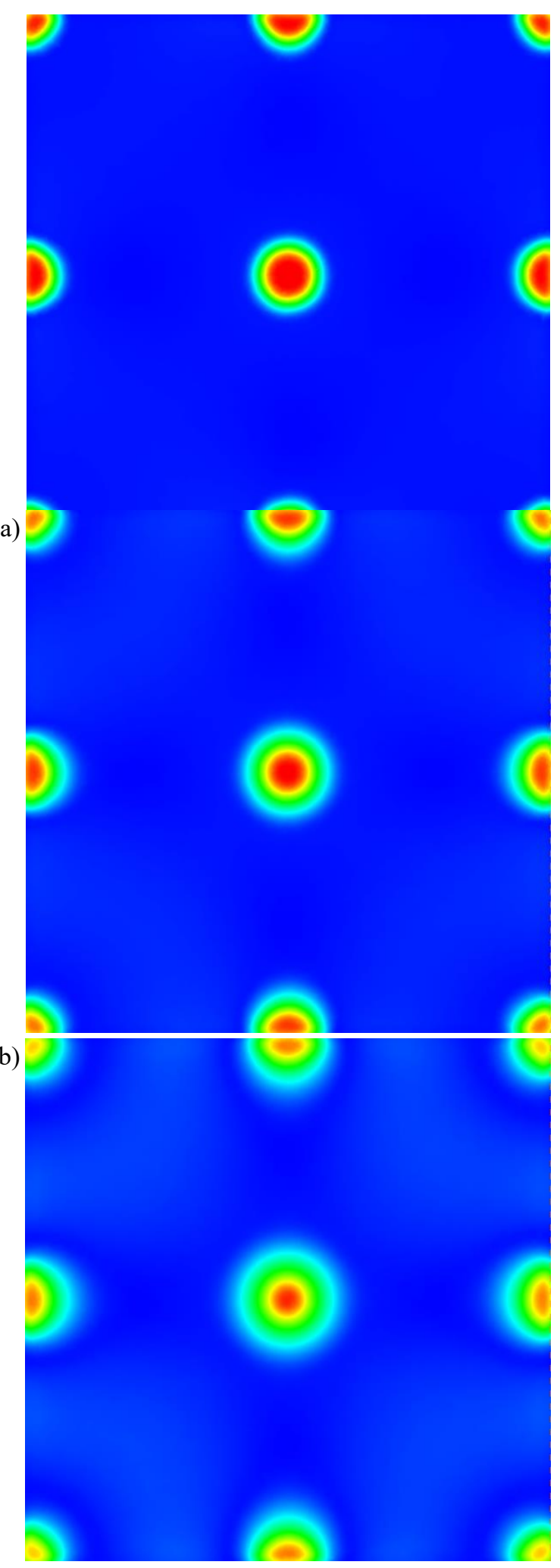

c)

Velocity[m/s]: $\quad \begin{array}{llllllllllllllll} & 0.00 & 0.36 & 0.71 & 1.07 & 1.43 & 1.79 & 2.14 & 2.50 & 2.86 & 3.21 & 3.57 & 3.93 & 4.29 & 4.64 & 5.00\end{array}$ 
d)

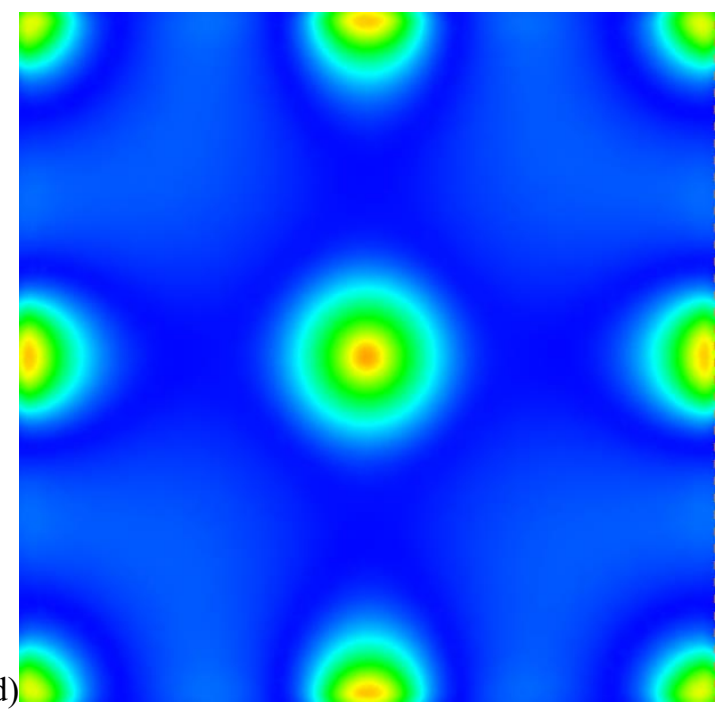

e)

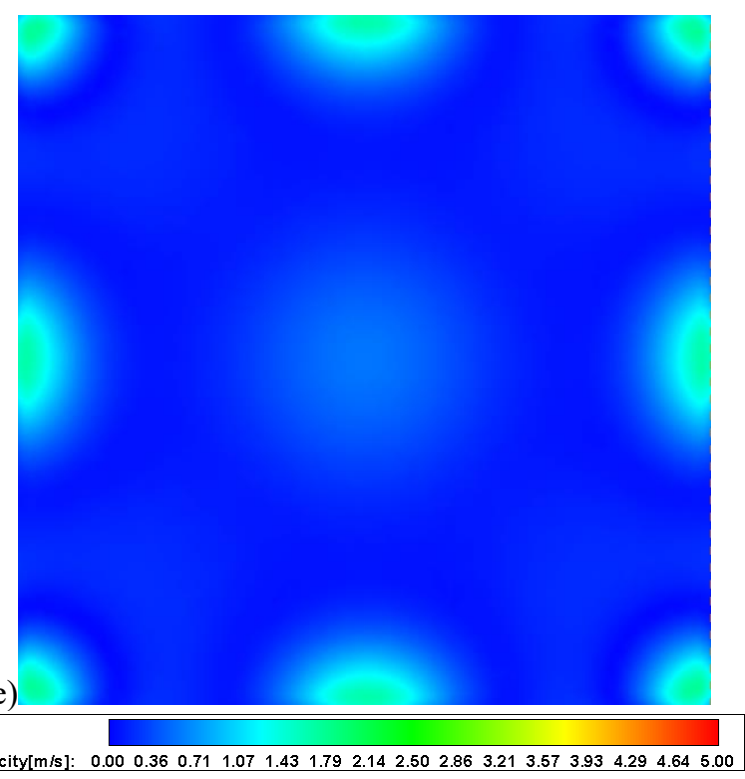

Fig. 16. Velocity fields for circular orifices at: a) $1 \mathrm{De}$;

b)2De; c)3De; d)4De; e)10De

Comparations of velocity profile from the numerical cases are presented in Figure 16, 17 and from the PIV measurements are presented in Figure 20. The comparations were made in frontal and sagittal planes. Figure 16 presents the evolution of the jets at different equivalent diameter (De) distance for the lobed diffuser, while Figure 17 presents the evolution of jets from the circular one. The equivalent diameter (De) distance, shown as frontal plane velocity field, were made at $1 \mathrm{De}$, 2De, 3De and 4De. The sagittal velocity fields comparations between the two approaches were made on a length of $30 \mathrm{~mm}$, approximately 4De (Figure 21 ). The velocity profiles that were compared were those centred on the orifice's axes (Figure 19).

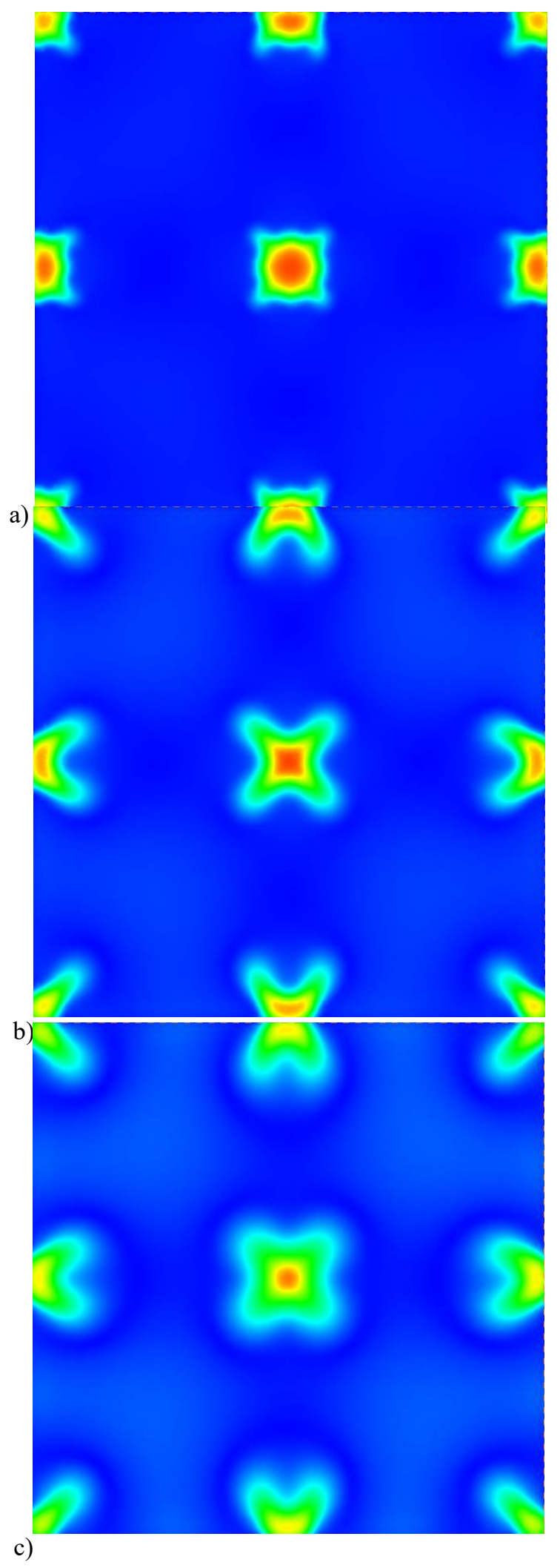

c) 


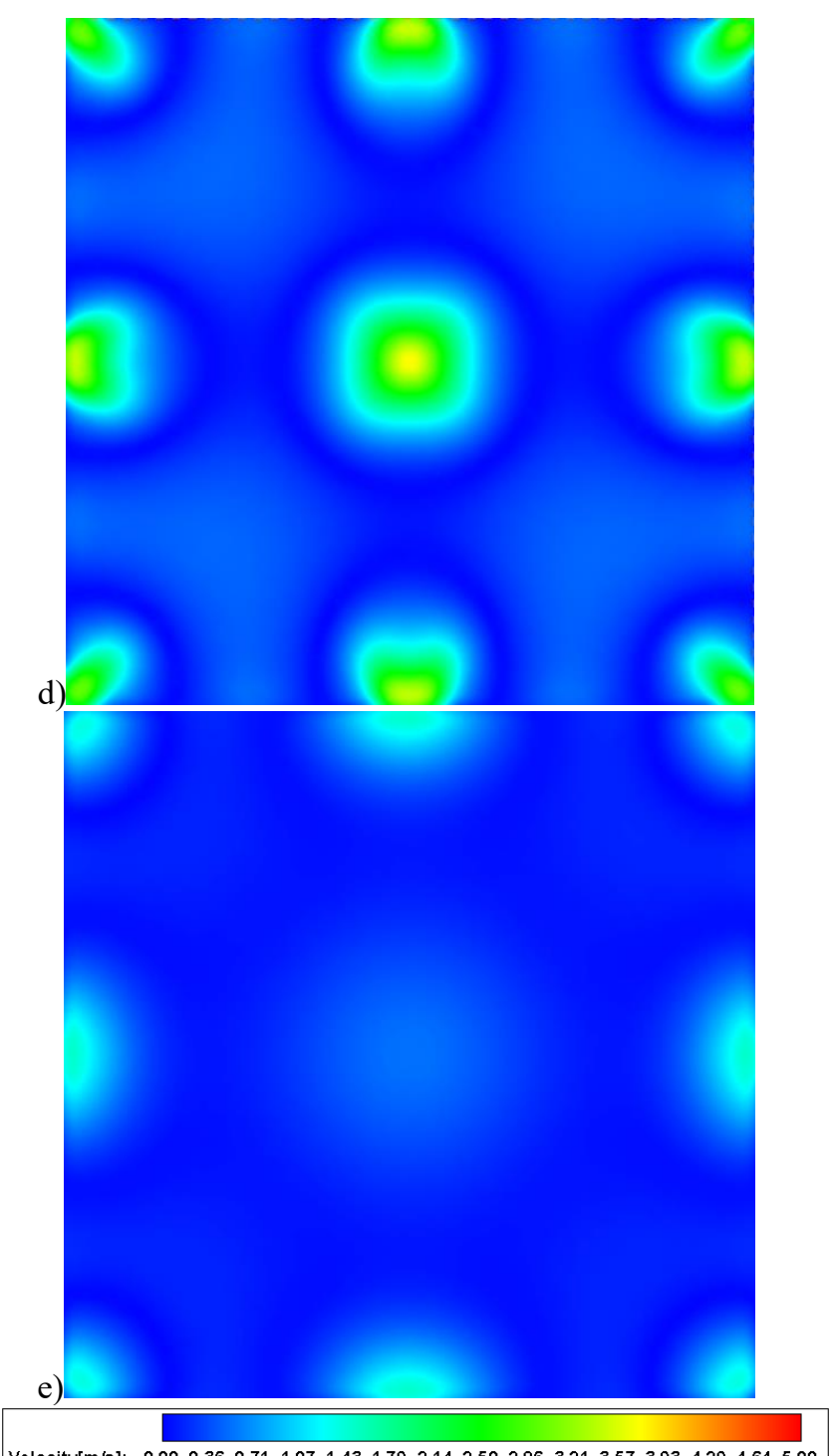

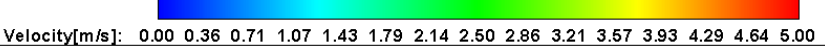

Fig. 17. Velocity fields for lobbed orifices at: a)1De; b)2De; c)3De; d)4De; e)10De

Isometric profiles of velocity fields from the two diffusers can be seen in Figure 18.

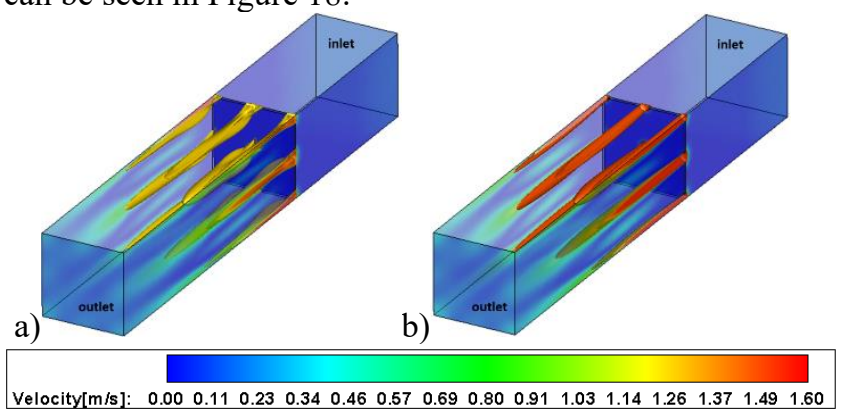

Fig. 18. The location of the velocity profiles comparations between the two orifices: a) lobbed; b) circular

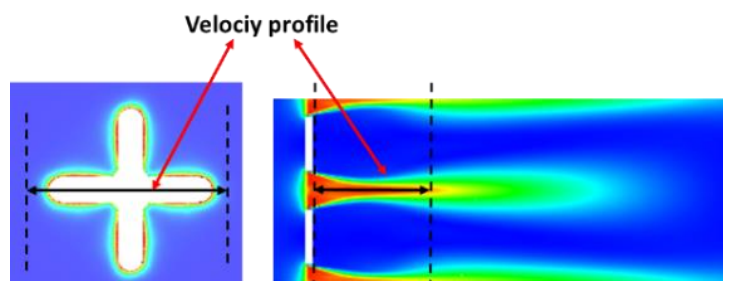

Fig. 19. Axes for velocity profiles comparison between the two approaches: left - front plane; right - sagital plane
Isometric views with the velocity fields resulted from the PIV measurements after post-processing them in Tecplot can be seen in Figure 20 from both diffusers, lobbed and circular.

a)

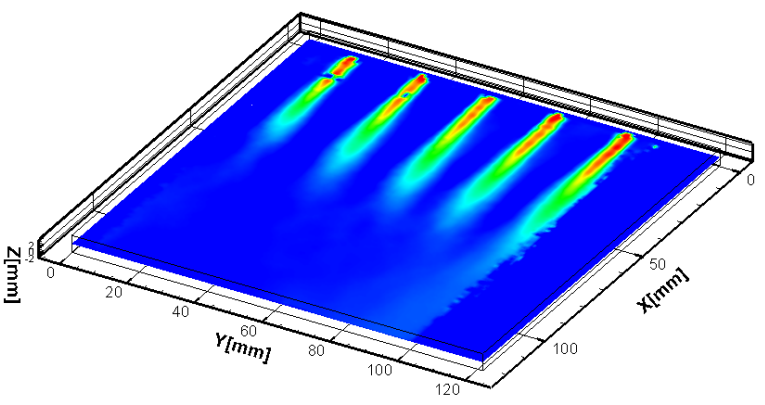

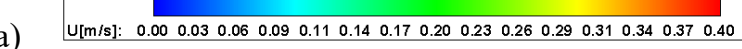

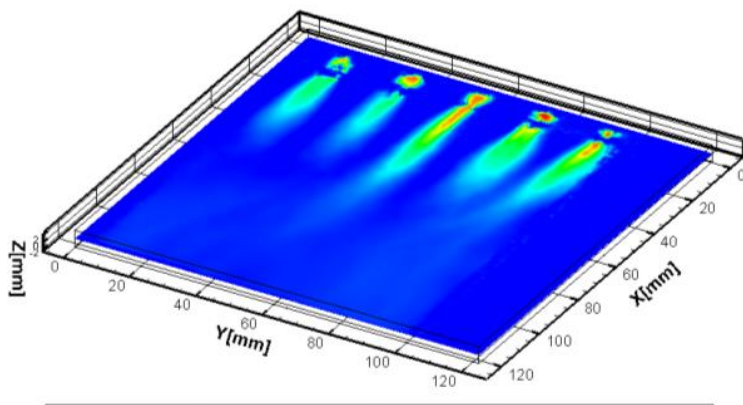

b)

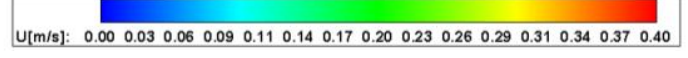

Fig. 20. Isometric views of the velocity fields resulted from the PIV measurements and from the post-processing in Tecplot: a) circular orifices; b) lobed orifices

A comparison of velocity profiles resulted from the two approaches was made and can be seen in Figure 21 and 22, according to the axes shown in Figure 19. Good agreement between the two approaches can be seen.

a)
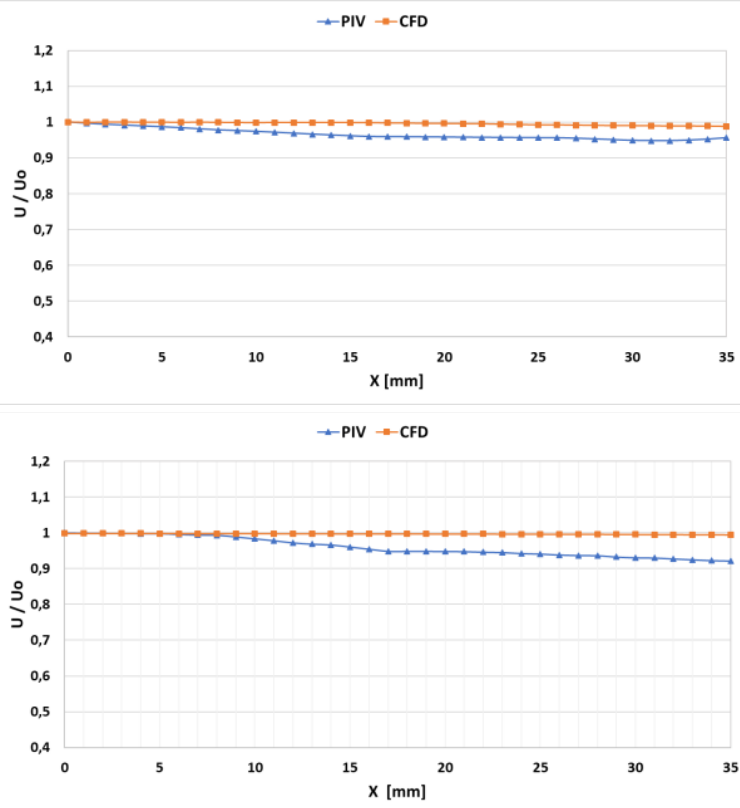

Fig. 21. Normalized longitudinal (sagittal) velocity profiles comparation for: a) lobbed orfice; b) circular orfice 


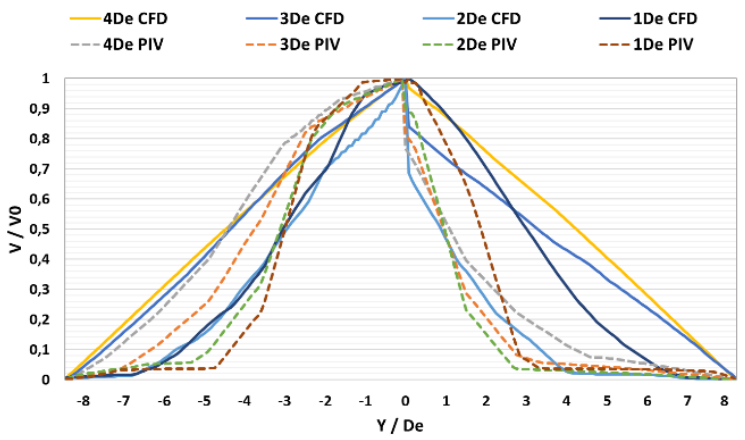

a)

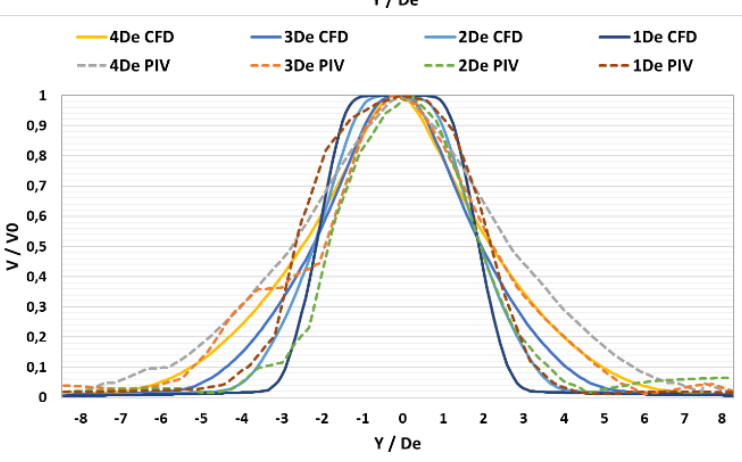

b)

Fig. 22. Normalized frontal, centered, velocity profiles comparation for: a) lobbed orfice; b) circular orfice

The slight discrepancies between the results are due to measurements errors and because the numerical model tends to achieve perfect results.

\section{Discussions and conclusions}

This study presents the analysis of two diffusers with different orifices, lobbed and circular, from two approaches, numerical and a PIV stereoscopic. Slight differences were seen between the results of the two approaches but with a good overall agreement. In addition to the different allure of the jets from the two orifices, which was expected to happen, it was observed that after 10 equivalent diameters (De) the velocity profiles tend to stabilize. The aim of this research was to create a numerical model from which we can take the results and use them as boundary conditions for other numerical studies, to validate the numerical model with experimental data, to analyse the interaction between the air jets through the orifices and to correlate the results with other studies in this field. It has been seen in this research and previous studies [3, 4] that the lobbed orifices can produce a larger mixing of the air compared to a circular orifices, aspect which would result in better air distribution due to the higher mixing. A better air distribution will directly influence the thermal stratification. Implementing lobbed orifices in the terminal unit can also influence the aerosol distribution and the energy consumption of the HVAC unit. There are studies that showed different technical aspects (pressure lose, noise studies) of an air terminal with different orifices [16], but future studies are needed in order to have a better understanding of their influence in a real scale.

\section{Acknowledgment}

This work was supported by the grant of the Romanian National Authority for Scientific Research, CNCS, UEFISCDI, PHANTOM - Passive flow control for heat and mass transfer enhancement of impinging jets PN-IIIP4-ID-PCE-2016-0758

\section{Bibliography}

1. Bennia, A., et al., Contribution to the Experimental and Numerical Dynamic Study of a Turbulent Jet Issued from Lobed Diffuser. Vol. 9. 2016. 2957-2967.

2. Bennia, A., et al., Experimental and numerical investigation of a turbulent lobed diffuser jet: Application to residential comfort. Vol. 19. 2018. 104.

3. Meslem, A., I. Nastase, and F. Allard, Passive mixing control for innovative air diffusion terminal devices for buildings. Building and Environment, 2010. 45: p. 2679-2688.

4. Nastase, I. and A. Meslem, Passive control of jet flows using lobed nozzle geometries. Vol. 8. 2007.

5. Dia, A., Simulation of lobed jet flows for optimisation of Air Diffusion Terminal Units. 2012, Université de La Rochelle.

6. Dimotakis, P.E., The mixing transition in turbulent flows. Journal of Fluid Mechanics, 2000. 409: p. 69-98.

7. Meslem, A., et al., A comparison of three turbulence models for the prediction of parallel lobed jets in perforated panel optimization. Building and Environment, 2011. 46: p. 2203-2219.

8. Meslem, A., et al., Optimization of Lobed Perforated Panel Diffuser: Numerical Study of Orifice Geometry. Vol. 6. 2012.

9. Nastase, I., A. Meslem, and P. Gervais, Primary and secondary vortical structures contribution in the entrainement of low Reynolds number jet flows. Experiments in Fluids, 2008. 44(6): p. 1027-1033.

10. Meslem, A., et al., Comparison of turbulence models in simulating jet flow from a cross-shaped orifice. European Journal of Mechanics - B/Fluids, 2014. 44: p. 100-120.

11. I, N., Analyse des jets lobés en vue de leur intégration dans les Unités Terminales de Diffusion d'air. 2007, Université de La Rochelle.

12. Nastase, I., A. Meslem, and M. El Hassan, Image processing analysis of vortex dynamics of lobed jets from three-dimensional diffusers. Vol. 43. 2011. 065502.

13. M. Belovich, V. and M. Samimy, Mixing Processes in a Coaxial Geometry with a Central Lobed MixerNozzle. Vol. 35. 1997. 838-841.

14. $\mathrm{Hu}, \mathrm{H}$., et al., PASSIVE CONTROL ON JET MIXING FLOWS BY USING VORTEX GENERATORS. 2000.

15. $\mathrm{Hu}, \mathrm{H}$., et al., Mixing Process in a Lobed Jet Flow. AIAA Journal, 2002. 40(7): p. 1339-1345.

16. Tacutu, L., et al., Real scale experimental study for performance evaluation of unidirectional air diffuser perforated panels. Vol. 32. 2018. 01014. 